\title{
Parental management of developmental coordination disorder: Validation of a French translation of the Parental Knowledge and Skills Questionnaire
}

\author{
Gabrielle Pratte ${ }^{1}$, Tibor Schuster ${ }^{2}$, Jade Berbari ${ }^{3}$, Chantal Camden*3,4 \\ ${ }^{1}$ Faculty of Medicine and Health Sciences, Sherbrooke University, Québec, Canada \\ ${ }^{2}$ Department of Family Medicine, McGill University, Québec, Canada \\ ${ }^{3}$ CHUS Research Center, Québec, Canada \\ ${ }^{4}$ School of Rehabilitation, Sherbrooke University, Québec, Canada
}

Received: March 7, 2019

DOI: $10.5430 /$ ijh.v5n2p36
Accepted: May 23, 2019

Online Published: June 10, 2019

URL: https://doi.org/10.5430/ijh.v5n2p36

\begin{abstract}
Background: Best practices in Developmental Coordination Disorder (DCD) recommend building parental capacities to enhance children's functioning but few outcome measures are available to document the impact of such interventions. The Parent Knowledge and Skills Questionnaire (PKSQ) is a valid instrument currently only available in English evaluating parental perception of their ability to manage their child, understand DCD's impact and use knowledge about DCD. The aim of the study was to translate the PKSQ in French and assess the factor structure consistency between the French and English versions.

Methods: PKSQ was translated in French using a back-translation method. Parents of children with DCD were recruited in collaboration with the DCD association and rehabilitation centers. Parents completed the questionnaire online $(\mathrm{n}=99)$. Confirmatory factor analysis was conducted based on the questionnaire response data and estimated item-domain correlations were compared with the English version.

Results: All items of the original PKSQ were culturally appropriate in the Quebec French context and were thus included in the final version $(n=22)$. The French questionnaire version showed overall good consistency with the English version. The frequency of statistically inferior item-domain correlations was consistent with the expected rate of 5\% under the no difference assumption (3/22, $95 \%$ confidence interval: $3 \%$ to $35 \%)$. Items showed moderate to large correlations with their underlying construct (estimated item-domain correlations between 0.45-0.91).

Conclusions: Results suggest the French version of the PKSQ is consistent with the 3-factor structure of the English version, and may now be used to evaluate interventions based on DCD best practices.
\end{abstract}

Key Words: Developmental coordination disorder, Parent Knowledge and Skills Questionnaire, Construct validity

\section{INTRODUCTION}

Developmental coordination disorder (DCD) is a chronic condition affecting the ability to plan and execute activities requiring motor coordination. ${ }^{[1]}$ DCD is prevalent and affects up to 5\%-6\% of children, according to the DSM-5. ${ }^{[1]}$ Children with DCD have difficulties performing everyday

\footnotetext{
*Correspondence: Chantal Camden; Email: Chantal.camden@usherbrooke.ca; Address: School of rehabilitation, Sherbrooke University, 3001, 12e avenue Nord, Sherbrooke (Québec), J1H 5N4, Canada.
} 
activities such as putting on clothes, learning sports and handwriting. ${ }^{[2,3]}$ In addition, children with DCD have lower quality of life compared to their peers ${ }^{[4]}$ and are at greater risk to develop depressive or anxious symptoms, ${ }^{[5,6]}$ cardiovascular disease $^{[7]}$ and obesity. ${ }^{[8,9]}$

To prevent or decrease the negative impacts of DCD on children's daily lives, best practices suggest supporting parents and helping them manage DCD. ${ }^{[10,11]}$ To build parental capacities, interventions should provide parents with information about DCD, increase their understanding of DCD's consequences on their child's daily life and help them better manage DCD. ${ }^{[12]}$ Despite a broad consensus about these best practices, few studies evaluating interventions aiming directly at supporting parents are available. ${ }^{[12-18]}$ Interventions such as Co-op certainly include capacity-building, ${ }^{[19]}$ but providing parents with information about DCD is not the core focus of the intervention. The few interventions centered on parental information include informational group sessions, ${ }^{[20]}$ online and DVD information modules ${ }^{[21,22]}$ or individual coaching to parents. ${ }^{[23]}$ Results of these interventions suggest parents appreciated receiving information and improved their ability to manage DCD, but the level of evidence is weak. Only one study conducted a pre-post evaluation to document an intervention's impact on parental knowledge and ability to manage DCD. ${ }^{[21]}$

The lack of validated outcome measures to evaluate the impact of interventions aiming to increase parental knowledge and skills is currently a barrier to design randomized controlled trials based on DCD best practices. To our knowledge, only one questionnaire, the Parents Knowledge and Skills Questionnaire (PKSQ), has been created to evaluate the effect of an intervention aiming at supporting parents of children with DCD. ${ }^{[24]}$ This questionnaire built on other questionnaires that were originally used to evaluate a DCD service delivery model ${ }^{[25]}$ and were further revised to evaluate a DCD parent module, ${ }^{[21]}$ but until recently, the PKSQ had never been validated. Recently, however, its construct validity has been evaluated within the context of a new service delivery model evaluation and a three-factors structure emerged: 1) Managing the child, 2) Understanding DCD's impact on the child and 3) Understanding and using knowledge about DCD. ${ }^{[24]}$ However, this questionnaire is only available in English and has never been validated in other contexts. Since no questionnaire is available in French to evaluate interventions aiming at supporting parents of children with DCD and increasing their knowledge and skills, a study was undertaken to translate the PKSQ in French and evaluate its validity by assessing factor structure consistency between the French and English versions.

\section{MeTHODS}

\subsection{Study design}

A back-translation process, based on Brislin' method, ${ }^{[26]}$ was used to translate the PKSQ in French. The English version of the PKSQ was first translated in French by a bilingual researcher. The newly translated French version was then translated back into English by a second bilingual researcher. Both researchers and a third bilingual referee met to compare the original and back-translated English versions of the PKSQ. Questions of clarifications were asked to the authors of the original PKSQ to ensure cultural fidelity of the translation. The third independent researcher validated final modifications.

The final French version was pretested with two French speaking adults independent of the translation team. The French version of the questionnaire was then uploaded online, on REDCap (Research Electronic Data Capture), ${ }^{[27]}$ an electronic data capture tool hosted at Sherbrooke University. Questionnaires were completed by parents in the context of a public consultation that aimed to determine research and knowledge translation priorities for DCD stakeholders. The research project was approved by the Ethics board of the CHUS research center (MP-31-2017-1467).

\subsection{Parent Knowledge and Skills Questionnaire}

The PKSQ includes 22 questions using a 7-point Likert scale ranging from "Not at all" to "To a very great extent". Validation of the questionnaire established a three-factor structure evaluating the following constructs: 1) Managing the child, 2) Understanding DCD's impact on the child, and 3) Understanding and using knowledge about DCD. ${ }^{[24]}$ Examples of questions include: "To what extent do you feel that you understand the impact of coordination difficulties on your child's ability to manage daily tasks at home (e.g., putting clothes on, eating, brushing teeth, bathing, getting ready to go to school)", and "To what extent do you feel confident in your ability to: Adapt the environment and tasks to meet your child's needs?".

\subsection{Study process}

Participants were recruited in the province of Quebec, Canada, via the provincial DCD association, two rehabilitation centers, social media and word-of-mouth, as part of a community-based research partnership. In addition to the PKSQ, participants were invited to complete sociodemographic questions (e.g., sex and age of the parents and children, co-occurring disorders). Participants had to confirm they were parents of a child with DCD to have access to the PKSQ questionnaire, since the public consultation also included other stakeholders (see Camden et al., ${ }^{[28]}$ for more details). 


\subsection{Analysis}

Frequency distributions were reported to describe demographic characteristics. Confirmatory factor analysis (CFA) was performed on PKSQ results using $\mathrm{R}$ version 3.5.1 [29] with Lavaan package version 0.6-3. CFA was done on the standardized scale of the item variables to enable better interpretation of estimated factor loadings i.e., estimating itemdomain correlation coefficients instead of regression model coefficients (slopes of the underlying structural equation model). We used English version results from the principal factor exploratory factor analysis (EFA) with orthogonal rotation to build the CFA model that set which questionnaireitem is represented on which factor. The factor variances were forced to be 1 and factors were allowed to be correlated with each other. Each pair of questionnaire-items was set to be non-correlated. Ninety-five percent confidence intervals for factor loadings were calculated with bootstrap percentile method using 1,000 repeated samples and constraint not to exceed 1. Factor loadings and associated 95\% confidence intervals were computed for each questionnaire-item and compared with English reference data (resulting from the same model CFA and same bootstrapping method) provided by the team who developed the original questionnaire. ${ }^{[24]}$ Items for which confidence intervals mutually included the factor loadings from both questionnaires (French and English version) were considered to be consistent. As $95 \%$ confidence intervals were used for the statistical comparison, a Type I error probability of 5\% was anticipated. ${ }^{[30]}$ Factor loadings around 0.1 were considered small, around 0.3 medium and around 0.5 large. ${ }^{[31]}$

\section{Results}

\subsection{Participants}

Ninety-nine parents were recruited in this study. Participants were mostly mothers $(95 \%)$ (see Table 1 ). Most of their children were between 5 and 12 years old $(81 \%), 4 \%$ of the respondents reported having preschool children, $13 \%$ having teenagers and $2 \%$ being the parent of an adult with DCD. In our sample, $94 \%$ of children with DCD had received professional services. Of those, $83 \%$ had received services in occupational therapy, $52 \%$ in speech language pathology, $35 \%$ in neuropsychology, $35 \%$ in physiotherapy and $32 \%$ in special education. Co-occurring disorders were reported by respondents for $77 \%$ of children. Among those who had a co-occurring disorder, diagnosis included attention deficit hyperactivity disorder (53\%), language disorder (25\%), anxiety disorder $(26 \%)$, sensory disorder $(22 \%)$, learning disability $(18 \%)$ or autism spectrum disorder $(6 \%)$.
Table 1. Participants demographic characteristics

\begin{tabular}{lcc}
\hline Characteristics & $\mathbf{N}^{*}$ & Count (\%) \\
\hline $\begin{array}{l}\text { Relation with the child } \\
\quad \quad \text { Mother }\end{array}$ & 98 & $93(95 \%)$ \\
$\begin{array}{l}\text { Number of children with DCD in the family } \\
\quad \quad \text { > } 1 \text { children with DCD }\end{array}$ & 96 & $11(11 \%)$ \\
$\begin{array}{l}\text { Child with DCD age } \\
\quad \quad \quad \text { Between 5-12 years old }\end{array}$ & 85 & $69(81 \%)$ \\
$\begin{array}{l}\text { Parent also having DCD } \\
\quad \quad \quad \text { Yes }\end{array}$ & 99 & $4(4 \%)$ \\
$\begin{array}{l}\text { Presence of co-occurring disorders } \\
\quad \quad \quad \text { Yes }\end{array}$ & 95 & $73(77 \%)$ \\
$\begin{array}{l}\text { Child received support services } \\
\quad \quad \quad \text { Yes }\end{array}$ & 96 & $90(94 \%)$ \\
\hline $\begin{array}{l}\text { Note. } \\
\text { data in the percentages presented, total N = 99 }\end{array}$ &
\end{tabular}

\subsection{Cross-cultural translation}

Most questionnaire items and examples were culturally relevant for the Quebec French version. Only a minor modification was made to one item, removing a concept since printing and handwriting are not differentiated in the everyday language in French. All other items and examples were kept as originally worded.

\subsection{Confirmatory factor analysis}

Estimated factor loadings from the CFA for the French and English versions of the questionnaire are presented in Figures 1, 2 and 3, based on the 3-factor model. For the first factor (Managing the child), all items showed item-domain correlations that were consistent in the two versions as the 95\% confidence intervals mutually included the respective point estimates for each item (see Figure 1). For question 16 , point estimates did not fall into the confidence intervals of the complement questionnaire (French CFA estimate $=$ 0.60 [0.41-0.77], English CFA estimate = 0.78 [0.65-0.90]). However, item-domain correlations of French items within this factor are mostly large $(\mathrm{CFA}=$ generally from 0.60 to 0.81). Only the estimated factor loading for question 20 was found to be below 0.5 , with relatively high imprecision (To what extent do you feel confident in your ability to: Find community activities that will support your child; $\mathrm{CFA}=$ $0.45[0.26-0.62])$.

All items of the second factor were highly correlated with the underlying construct, "understanding the impact of DCD on the child" (CFA from 0.72 to 0.91) (see Figure 2). For this construct, mostly all French point estimates were comprised in English confidence intervals and vice versa.

For the third factor ("Understanding and using knowledge about DCD”), all French items point estimates represented large correlations with the construct (CFA item-domain cor- 
relations from 0.58 to 0.88 ) (see Figure 3). All estimated For question 10, the French version estimate (CFA factor factor loadings of the French version, except one, fall into loading $=0.81$ [0.62-0.98]) was superior to the correspondconfidence intervals of the English version and conversely. ing estimate from the English version (CFA loading $=0.55$ [0.39-0.69]).

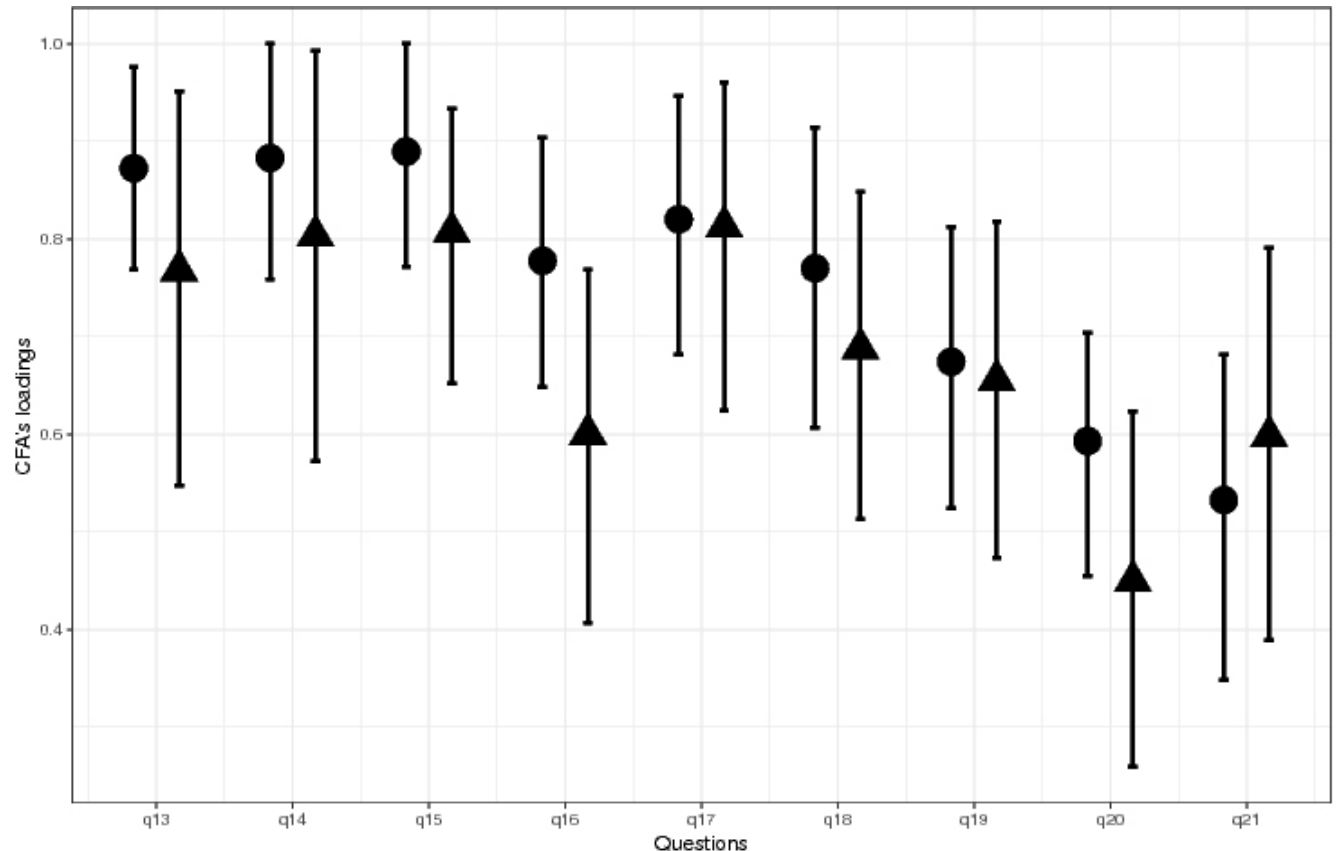

Figure 1. Comparison of English and French factor loadings based on the CFA for factor 1 (Managing the child) Circle $=$ English loading on the CFA; Triangle $=$ French loading on the CFA

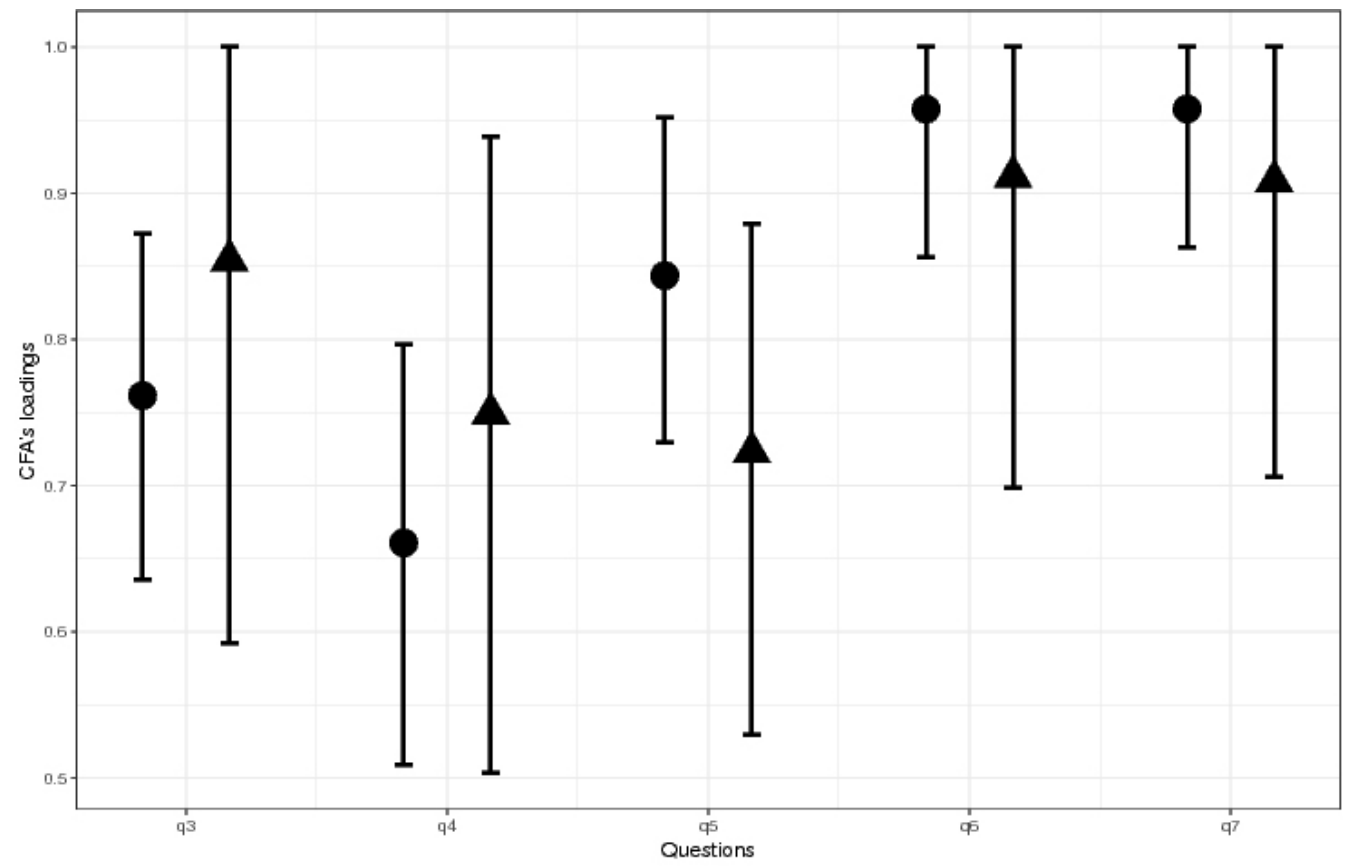

Figure 2. Comparison of English and French factor loadings based on the CFA for factor 2 (understanding the impact of DCD on the child)

Circle $=$ English loading on the CFA; Triangle $=$ French loading on the CFA 


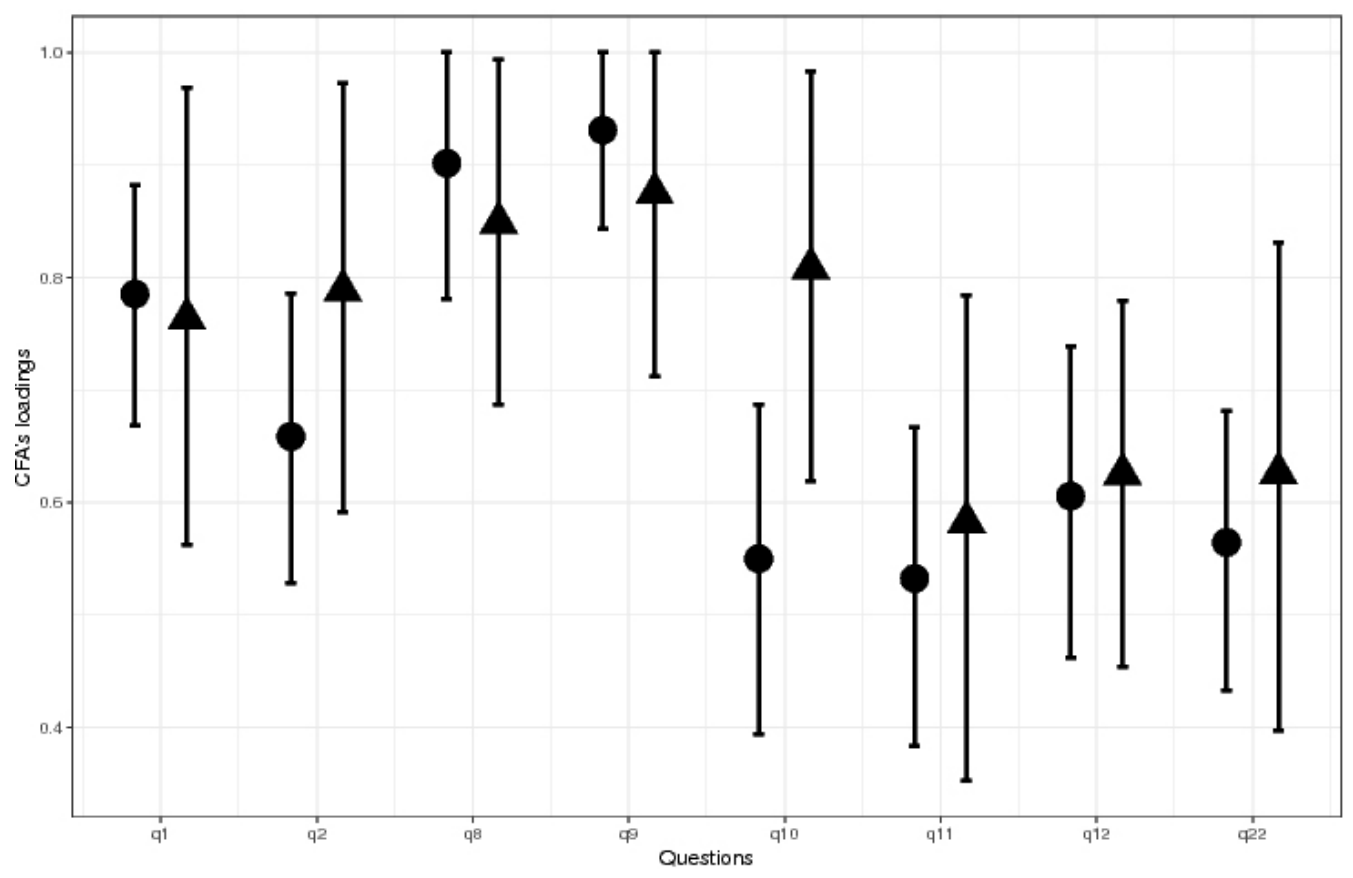

Figure 3. Comparison of English and French factor loadings based on the CFA for factor 3 (Understanding and using knowledge about DCD)

Circle $=$ English loading on the CFA; Triangle $=$ French loading on the CFA

\section{Discussion}

The aim of this paper was to validate a French version of the PKSQ by assessing the consistency of factor structures between the French and English versions. Globally, results show that overall correspondence of French and English items is good. ${ }^{[24,30]}$ Pre-tests on back-translated PKSQ did not raise any cultural concerns or confusions on item meaning. It is important to note that the original English version of the questionnaire was developed in Ontario, Canada, ${ }^{[24]}$ which is a similar culture to the context in which the French translation has been validated. These findings suggest the PKSQ might be applicable to various contexts, especially in Western countries with similar cultures.

As most confidence intervals for item-domain correlations mutually included the point estimates of the French and English version, the factor structures were found to be statistically consistent. The French PKSQ is consistent with the strong 3-factors model previously found for the English version; the three same constructs were thus kept in the French version: 1- Managing the child, 2- Understanding DCD's impact on the child and 3- Understanding and using knowledge about DCD. ${ }^{[24]}$

Only 3 items did not meet the statistical criterion for consistency of factor loading estimates. Of these 3 items, question 10 showed a higher correlation with its construct in the French questionnaire than in the English version. With a total of 22 items being compared and 3 observed inconsistencies, the possible relative frequency of substantial deviations is between 3\% and 35\% (exact 95\% confidence interval for a proportion) and therefore still consistent with an anticipated Type I error rate of 5\%. ${ }^{[30]}$ The only item for which the French estimate clearly fell under the English confidence interval was question 16 ("To what extent do you feel confident in your ability to: Manage the day-to-day stresses associated with being a parent of a child with motor challenges?"). Considering the nature of the data collection context (i.e., public consultation about DCD research and knowledge translation priorities), parents in our sample might represent a more empowered group who had already received more support, thus having more resources to manage their stress, compared to the parents surveyed for the validation of the original questionnaire whose child had just been identified as potentially having DCD in the context of a school-based intervention. ${ }^{[24,32]}$

All items except one had a large correlation with its associate construct. Question 20 ("Find community activities that will support your child?") showed a moderate correlation. Interestingly, it is also one of the items in the English version that had the lowest correlation with its construct. This finding could be explained by the fact item 20 relates to system navigation, which is documented as an important issue for most families of children with DCD. ${ }^{[13,33]}$ System navigation might be a confounding construct affecting its correlation 
with the construct of Understanding and using knowledge about DCD in both the French and English versions.

However, because of the complementarity of the information brought by this question, we have decided to keep this item in the French version of the questionnaire. Moreover, the itemdomain correlation estimates of all items remain medium or large, thus indicating a fairly good construct validity of the questionnaire. ${ }^{[30]}$

The three constructs identified are largely supported by DCD best practices. ${ }^{[10]}$ Construct one (Managing the child) closely relates to building capacity, where parents are perceived to be key team players and the ones who might have the greatest impact on their child's functioning. ${ }^{[10]}$ It is important to note that in the PKSQ, this construct goes beyond DCD since parents are asked about their perceptions of managing their child's condition and dealing with motor difficulties in general. Although the PKSQ has only been used thus far with a DCD population, items pertaining to construct one might even be applicable with other populations having mild motor difficulties, where building parental capacities to have parents managing their child is also part of best practices. ${ }^{[34]}$ This would however warrant more investigation. Constructs 2 (Understanding the impact of DCD on the child) and 3 (Understanding and using knowledge about DCD) are more specific to the DCD population and best practices. They both relate to providing information to parents, so they better understand the condition and are able to adapt to the child's needs and advocate for him. ${ }^{[13,15,35]}$ The PKSQ thus included all important concepts related to DCD best practices about parental knowledge and skills to manage $\mathrm{DCD}^{[10]}$ and might thus be an important tool to evaluate evidenceinformed intervention for parents.

The principal limit of this study is the relatively small sample size, known to have an impact on confidence interval width and therefore on the precision of the estimated factor loadings. However, most item confidence intervals were comparable in English and French versions, despite a larger sample size in the English validation sample. We employed a statistical approach for comparing factor loadings on an "absence of evidence supports" base. Our findings therefore must be interpreted with caution as they indicate consistency but do not, without further assumptions, allow for conclusion of strict equivalence. Larger confirmatory studies should be conducted to enable multiplicity-adjusted comparisons of item-domain correlations while taking clinically relevant equivalence margins into account. A major strength of this study is the sample representativeness of the DCD population included. For instance, a high percentage of our sample had a co-occurring disorder, representing the well documented presence of a variety of co-occurring disorders in the DCD population. ${ }^{[1,10,36-38]}$ Our sample representativeness ensures generalizability of the results in most DCD populations and contexts in which PKSQ could be used.

\section{Conclusion}

This validation study is a first step for evaluating interventions based on best practices and aiming to build parental capacity to manage DCD. Our results demonstrate the PKSQ might be a valid tool for parents of children with DCD in Western countries and established the construct validity of its French version. The French version is an important asset for research on families of children with DCD and for the development of evidence-based interventions for French speaking parents. To complete this work, future studies could explore test-retest fidelity of the PKSQ and its applicability in a broad range of contexts.

\section{FUNDING}

This study was financed by the Canadian Institutes of Health Research (grant number: 360634) and the University of Sherbrooke.

\section{ACKNOWLEDGEMENTS}

Authors would like to acknowledge the work of Catherine Allard, our biostatistician. We also wish to thank the Partnering for Change team who developed and validated the English version of the PKSQ, including Wenonah Campbell, Cindy DeCola, Leah Dix and Scott Veldhuizen. This study was financed by the Canadian Institutes of Health Research (grant number: 360634) and the University of Sherbrooke.

\section{CONFlicts OF INTEREST Disclosure}

The authors declare no conflicts of interest.

\section{REFERENCES}

[1] American Psychiatric Association. Desk Reference to the Diagnostic Criteria From DSM-5®. American Psychiatric Publishing; 2014.

[2] Jasmin E, Tetreault S, Joly J. Ecosystemic needs assessment for children with developmental coordination disorder in elementary school: multiple case studies. Physical \& Occupational Therapy in Pediatrics. 2014; 34(4): 424-442. PMid:24649977. https ://doi.org/10.3 $109 / 01942638.2014 .899284$

[3] Van der Linde BW, van Netten JJ, Otten B, et al. Activities of Daily Living in Children With Developmental Coordination Dis- 
order: Performance, Learning, and Participation. Physical Therapy. 2015; 95(11): 1496-1506. PMid:26045605. https://doi .org/10 $.2522 / \mathrm{ptj} .20140211$

[4] Zwicker JG, Suto M, Harris SR, et al. Developmental coordination disorder is more than a motor problem: Children describe the impact of daily struggles on their quality of life. British Journal of Occupational Therapy. 2018; 81(2): 65-73. https://doi.org/10.1177/ 0308022617735046

[5] Fuhrmann P, Equit M, Schmidt K, et al. Prevalence of depressive symptoms and associated developmental disorders in preschool children: a population-based study. European Child \& Adolescent Psychiatry. 2014; 23(4): 219-224. PMid:23839066. https: //doi.org/10.1007/s00787-013-0452-4

[6] Missiuna C, Cairney J, Pollock N, et al. Psychological distress in children with developmental coordination disorder and attention-deficit hyperactivity disorder. Research in Developmental Disabilities. 2014; 35(5): 1198-1207. PMid:24559609. https://doi.org/10.1016/ j.ridd.2014.01.007

[7] Philips NE, Chirico D, Cairney J, et al. Arterial stiffness in children with and without probable developmental coordination disorder. Research in Developmental Disabilities. 2016; 59: 138-146. PMid:27552249. https://doi.org/10.1016/j.ridd.2016.07 .011

[8] Cermak SA, Katz N, Weintraub N, et al. Participation in physical activity, fitness, and risk for obesity in children with developmental coordination disorder: A cross-cultural study. Occupational Therapy International. 2015; 22(4): 163-173. PMid:26123622. https://doi.org/10.1002/oti.1393

[9] Zhu YC, Cairney J, Li YC, et al. High risk for obesity in children with a subtype of developmental coordination disorder. Research in Developmental Disabilities. 2014; 35(7): 1727-1733. PMid:24685095. https://doi.org/10.1016/j.ridd.2014.02.020

[10] Blank R, Smits-Engelsman B, Polatajko H, et al. European Academy for Childhood Disability (EACD): Recommendations on the definition, diagnosis and intervention of developmental coordination disorder (long version)*. Developmental Medicine \& Child Neurology. 2012; 54(1): 54-93. PMid:22171930. https ://doi .org/10 $.1111 / \mathrm{j} .1469-8749.2011 .04171 . \mathrm{x}$

[11] Camden C, Wilson B, Kirby A, et al. Best practice principles for management of children with developmental coordination disorder (DCD): results of a scoping review. Child: Care, Health and Development. 2015; 41(1): 147-159. PMid:24387638. https : //doi.org/10.1111/cch.12128

[12] Morgan R, Long T. The Effectiveness of Occupational Therapy for Children with Developmental Coordination Disorder: A Review of the Qualitative Literature. British Journal of Occupational Therapy. 2012; 75(1): 10-18. https://doi.org/10.4276/030802212X13 261082051337

[13] Missiuna C, Moll S, Law M, et al. Mysteries and mazes: Parents' experiences of children with developmental coordination disorder. Canadian Journal of Occupational Therapy. 2006; 73(1): 7-17. PMid:16570837. https://doi.org/10.2182/cjot.05.0010

[14] Maciver D, Owen C, Flannery K, et al. Services for children with developmental co-ordination disorder: the experiences of parents. Child: Care, Health and Development. 2011; 37(3): 422-429. PMid:21276034. https://doi.org/10.1111/j.1365-2214.20 10.01197.x

[15] Novak C, Lingam R, Coad J, et al. "Providing more scaffolding": parenting a child with developmental co-ordination disorder, a hidden disability. Child: Care, Health \& Development. 2012; 38(6): 829-835. PMid:21848938. https://doi.org/10.1111/j.1365-2214.20 $11.01302 . x$
[16] Jasmin E, Tetreault S, Lariviere N, et al. Participation and needs of children with developmental coordination disorder at home and in the community: Perceptions of children and parents. Research in Developmental Disabilities. 2018; 73: 1-13. PMid:29245043. https://doi.org/10.1016/j.ridd.2017.12.011

[17] Alonso Soriano C, Hill EL, Crane L. Surveying parental experiences of receiving a diagnosis of developmental coordination disorder (DCD). Research in Developmental Disabilities. 2015; 43-44: 11-20. PMid:26151439. https://doi.org/10.1016/j.ridd.2 015.06 .001

[18] Armitage S, Swallow V, Kolehmainen N. Ingredients and change processes in occupational therapy for children: a grounded theory study. Scandinavian Journal of Occupational Therapy. 2017; 24(3): 208-213. PMid:27454899. https://doi .org/10.1080/110381 28.2016 .1201141

[19] Polatajko HJ, Mandich A. Enabling Occupation in Children: The Cognitive Orientation to Daily Occupational Performance (CO-OP) Approach. CAOT Publications ACE; 2004.

[20] Camden C, Léger F, Morel J, et al. A Service Delivery Model for Children with DCD Based on Principles of Best Practice. Physical \& Occupational Therapy in Pediatrics. 2015; 35(4): 412-425. PMid:25397664. https://doi.org/10.3109/01942638.2014. 978932

[21] Camden C, Foley V, Anaby D, et al. Using an evidence-based online module to improve parents' ability to support their child with Developmental Coordination Disorder. Disability and Health Journal. 2016; 9(3): 406-415. PMid:27155793. https://doi .org/10.101 $6 / j . d h j 0.2016 .04 .002$

[22] Miyahara M, Butson R, Cutfield R, et al. A pilot study of familyfocused tele-intervention for children with developmental coordination disorder: development and lessons learned. Telemedicine Journal and E-Health: The Official Journal of the American Telemedicine Association. 2009; 15(7): 707-712. PMid:19694593. https://doi . org/10.1089/tmj . 2009.0022

[23] Sugden DA, Chambers ME. Intervention in children with Developmental Coordination Disorder: The role of parents and teachers. British Journal of Educational Psychology. 2003; 73(4): 545-561. PMid:14713377. https://doi.org/10.1348/00070990332259 1235

[24] Campbell W, Camden C, DeCola C, et al. Building capacity. In: Partnering for change. C. Missiuna \& L. Rivard. CanChild: McMaster University; In preparation.

[25] Missiuna C, Pollock N, Campbell WN, et al. Use of the Medical Research Council Framework to develop a complex intervention in pediatric occupational therapy: Assessing feasibility. Research in Developmental Disabilities. 2012; 33(5): 1443-1452. PMid:22522203. https://doi.org/10.1016/j.ridd.2012.03.018

[26] Richard WB. The wording and translation of research instruments. In: Field methods in cross-cultural research. 1986. p. 137-164. Available from: http://www. business.illinois.edu/shavit t/BA_531/brislin_wording.pdf

[27] Paul AH, Robert T, Robert T, et al. Research electronic data capture (REDCap) - A metadata-driven methodology and workflow process for providing translational research informatics support. Journal of Biomedical Informatics. 2009; 42(2): 377-381. PMid:18929686. https://doi.org/10.1016/j.jbi.2008.08.010

[28] Camden C, Meziane S, Maltais DB, et al. Supporting children in school and improving services are the research and knowledge transfer priorities for multiple stakeholders involved in Developmental Coordination Disorder. Health Expectations. Forthcoming 2019. 
[29] R Core Team. R: A language and environment for statistical computing. Vienna, Austria: R Foundation for Statistical Computing; 2018.

[30] Carter RE, Lubinsky J, Domholdt E. Rehabilitation Research: Principles and Applications. Elsevier Saunders; 2011.

[31] Cohen J. Statistical power analysis for the behavioral sciences. 1988.

[32] CanChild. Description of the Children - Partenering for change. CanChild; 2015. Findings from P4C. Available from: https://www.pa rtneringforchange.ca/findings/children/

[33] Stephenson E, Chesson R. 'Always the guiding hand': parents' accounts of the long-term implications of developmental co-ordination disorder for their children and families. Child: Care, Health and Development. 2008; 34(3): 335-343. PMid:18410640. https ://do i. org/10.1111/j.1365-2214.2007.00805.x

[34] Hughes AJ, Redsell SA, Glazebrook C. Motor Development Interventions for Preterm Infants: A Systematic Review and Meta-analysis. Pediatrics. 2016; 138(4): e20160147-e20160147. PMid:27638931. https ://doi.org/10.1542/peds.2016-0147
[35] Alsem MW, Ausems F, Verhoef M, et al. Information seeking by parents of children with physical disabilities: An exploratory qualitative study. Research in Developmental Disabilities. 2017; 60: 125-134. PMid:27914304. https://doi.org/10.1016/j.ridd.2016.11 .015

[36] Crane L, Sumner E, Hill EL. Emotional and behavioural problems in children with Developmental Coordination Disorder: Exploring parent and teacher reports. Research in Developmental Disabilities. 2017; 70: 67-74. PMid:28915470. https://doi.org/10.1016/ j.ridd.2017.08.001

[37] Flapper BCT, Schoemaker MM. Developmental coordination disorder in children with specific language impairment: Co-morbidity and impact on quality of life. Research in Developmental Disabilities. 2013; 34(2): 756-763. PMid:23220052. https://doi.org/10.1 016/j.ridd.2012.10.014

[38] Lingam R, Golding J, Jongmans MJ, et al. The association between developmental coordination disorder and other developmental traits. Pediatrics. 2010; 126(5): e1109-1118. PMid:20956425. https://doi.org/10.1542/peds. 2009-2789 